J. Korean Math. Soc. 51 (2014), No. 6, pp. 1305-1319

http://dx.doi.org/10.4134/JKMS.2014.51.6.1305

\title{
CONEAT SUBMODULES AND CONEAT-FLAT MODULES
}

\author{
ENGIN BÜYÜKAŞIK AND YILMAZ DURĞUN
}

\begin{abstract}
A submodule $N$ of a right $R$-module $M$ is called coneat if for every simple right $R$-module $S$, any homomorphism $N \rightarrow S$ can be extended to a homomorphism $M \rightarrow S$. $M$ is called coneat-flat if the kernel of any epimorphism $Y \rightarrow M \rightarrow 0$ is coneat in $Y$. It is proven that (1) coneat submodules of any right $R$-module are coclosed if and only if $R$ is right $K$-ring; (2) every right $R$-module is coneat-flat if and only if $R$ is right $V$-ring; (3) coneat submodules of right injective modules are exactly the modules which have no maximal submodules if and only if $R$ is right small ring. If $R$ is commutative, then a module $M$ is coneatflat if and only if $M^{+}$is $m$-injective. Every maximal left ideal of $R$ is finitely generated if and only if every absolutely pure left $R$-module is $m$ injective. A commutative ring $R$ is perfect if and only if every coneat-flat module is projective. We also study the rings over which coneat-flat and flat modules coincide.
\end{abstract}

\section{Introduction}

A subgroup $A$ of an abelian group $B$ is said to be neat in $B$ if $p A=A \cap p B$ for every prime integer $p$. The notion of neat subgroup was generalized to modules by Renault (see, [12]). Namely, a submodule $N$ of a right $R$-module $M$ is called neat in $M$, if for every simple right $R$-module $S, \operatorname{Hom}(S, M) \rightarrow$ $\operatorname{Hom}(S, M / N) \rightarrow 0$ is epic. Dually, in [8], a submodule $N$ of a right $R$-module $M$ is called coneat in $M$ if $\operatorname{Hom}(M, S) \rightarrow \operatorname{Hom}(N, S) \rightarrow 0$ is epic for every simple right $R$-module $S$. The notions of neat and coneat are coincide over the ring of integers. By [8, Theorem], the commutative domains over which neat and coneat submodules coincide are exactly the domains with finitely generated maximal ideals (i.e., N-domains). This result was extended to certain commutative rings in [5]. Recently, modules related to neat and coneat submodules are considered by several authors. In [5], a right $R$-module $M$ is called absolutely neat (resp. coneat) if $M$ is a neat (resp. coneat) submodule of any module containing it. According to [16], a right $R$-module $M$ is $m$-injective

Received March 31, 2014

2010 Mathematics Subject Classification. 16D10, 16D40, 16D80, 16E30.

Key words and phrases. neat submodule, coclosed submodule, coneat submodule, coneatflat module, absolutely neat module. 
if for any maximal right ideal $I$ of $R$, any homomorphism $I \rightarrow M$ can be extended to a homomorphism $R \rightarrow M$. By Theorem 3.4, a right $R$-module $M$ is absolutely neat if and only if $M$ is $m$-injective.

A ring $R$ is called right $C$-ring if $\operatorname{Soc}(R / I) \neq 0$ for each proper essential right ideal $I$ of $R$. Left perfect rings, right semiartinian rings and almost perfect domains are right $C$-rings.

A dual notion of $m$-injective modules has been studied in [1] and [2]. A module $M$ is called neat-flat if the kernel of any epimorphism $F \rightarrow M \rightarrow 0$ is a neat submodule of $F$. Closed submodules of any right $R$-module are neat, and neat submodules of any right $R$-module are closed if and only if $R$ is a right $C$-ring (see, [9, Theorem 5]). In [21], a module $M$ is called weak-flat if the kernel of any epimorphism $F \rightarrow M \rightarrow 0$ is a closed submodule of $F$. Hence, summing up we get, $R$ is a right $C$-ring if and only if every neat-flat right $R$-module is weak-flat.

We call $M$ coneat-flat if the kernel of any epimorphism $Y \rightarrow M \rightarrow 0$ is coneat in $Y$. In this paper, several characterizations of coneat submodules and coneat-flat modules are given. Some known results are generalized, and relations between coneat-flat modules and flat, $m$-injective, absolutely pure and projective modules are studied.

In Section 2, it is shown that a submodule $N$ of a right $R$-module $M$ is coneat if and only if for every maximal submodule $K$ of $N, N / K$ is a direct summand of $M / K$. A ring $R$ is a right $V$-ring if and only if submodules of right $R$-modules are coneat. $R$ is right small if and only if its absolutely coneat right modules are precisely those modules $M$ such that $M=\operatorname{Rad}(M)$.

In Section 3, we prove that, a module $M$ is coneat-flat if and only if $M \cong$ $P / N$ where $P$ is a projective $R$-module and $N$ is a coneat submodule of $P$. An $R$-module $M$ is coneat-flat if and only if and only if $M^{+}$is $m$-injective, over commutative rings. $R$ is a right $V$-ring if and only if every right $R$-module is coneat-flat.

In Section 4, we prove that, if $R$ is a left $C$-ring, then a right $R$-module $M$ is flat if and only if $\operatorname{Tor}_{1}^{R}(M, S)=0$ for each simple left $R$-module $S$. If $R$ is a commutative $C$-ring, then coneat-flat modules are only the flat modules, and the converse holds when $R$ is noetherian. $R$ is a left $N$-ring (i.e., maximal left ideals are finitely generated) if and only if every absolutely pure module is $m$-injective. A ring $R$ is left artinian if and only if $m$-injective left $R$-modules are precisely those modules $M$ with $M^{+}$is projective.

In Section 5, we consider the projectivity of coneat-flat modules. We show that, if $R$ is right perfect, then every coneat-flat $R$-module is projective, the converse hold if $R$ is commutative. Finitely presented coneat-flat modules are projective, over semiperfect rings and over commutative rings.

Throughout, $R$ is a ring with an identity element and all modules are unital right $R$-modules, unless otherwise stated. For an $R$-module $M$, the character module $\operatorname{Hom}_{\mathbb{Z}}(M, \mathbb{Q} / \mathbb{Z})$ is denoted by $M^{+}$. We use the notation $E(M)$, 
$\operatorname{Soc}(M), \operatorname{Rad}(M)$, for the injective hull, socle, radical of $M$ respectively. By $N \leq M$, we mean that $N$ is a submodule of $M$.

\section{Characterization and closure properties of coneat submodules}

In this section, several characterizations and some properties of coneat submodules are given. Recall that a submodule $K$ of $M$ is called small in $M$ (denoted by $K \ll M)$ if $M \neq K+T$ for every proper submodule $T$ of $M$. A submodule $L \leq M$ is called coclosed in $M$ if $L / N \ll M / N$ implies $L=N$ for every $N \leq L$.

Proposition 2.1. For a submodule $N \leq M$ the following are equivalent.

(1) $N$ is coneat in $M$.

(2) If $K \leq N$ with $N / K$ finitely generated and $N / K \ll M / K$, then $K=$ $N$.

(3) For any maximal submodule $K$ of $N, N / K$ is a direct summand of $M / K$.

(4) If $K$ is a maximal submodule of $N$, then there exists a maximal submodule $L$ of $M$ such that $K=N \cap L$.

Proof. (1) $\Rightarrow(4)$ Let $K$ be a maximal submodule of $N$ and $\pi: N \rightarrow N / K$ be the canonical epimorphism. By the hypothesis, there exists a homomorphism $f: M \rightarrow N / K$ such that $\left.f\right|_{N}=\pi$. Then Ker $f$ is a maximal submodule of $M$ and $N+\operatorname{Ker} f=M$. So that $N \cap \operatorname{Ker} f$ is a maximal submodule of $N$. Then $\pi(N \cap \operatorname{Ker} f)=f(N \cap \operatorname{Ker} f)=0$. Therefore $K=N \cap \operatorname{Ker} f$.

$(3) \Rightarrow(1)$ Let $S$ be a simple right $R$-module and $f: N \rightarrow S$ a nonzero homomorphism. Since $f$ is an epimorphism, without loss of generality we may assume that $S=N / K$ for some maximal submodule $K$ of $N$. So that Ker $f$ is a maximal submodule of $N$. Then, by (3), $M / \operatorname{Ker} f=(N / \operatorname{Ker} f) \oplus(L / \operatorname{Ker} f)$ for some $L \leq M$. Let $\tilde{f}: N / \operatorname{Ker} f \rightarrow N / K$ be the isomorphism induced by $f$. Consider the canonical epimorphisms $\pi: M \rightarrow M / \operatorname{Ker} f$ and $\pi^{\prime}: M / \operatorname{Ker} f \rightarrow$ $N / \operatorname{Ker} f$. Then the homomorphism $g=\tilde{f} \pi^{\prime} \pi$ is the extension of $f$.

$(2) \Rightarrow(3)$ is clear.

$(3) \Rightarrow(2)$ Suppose $N / K$ is finitely generated and $N / K \ll M / K$ for some proper submodule $K \leq N$. Then there is a maximal submodule $T$ of $N$ such that $K \leq T$ and $N / T \ll M / T$, because $N / T$ is the image of $N / K$ under the canonical epimorphism $f: M / K \rightarrow M / T$, a contradiction.

$(3) \Leftrightarrow(4)$ is straight forward.

Properties of coclosed modules in $[4,3.7]$ are adapted to coneat submodules as follows. The proof is omitted.

Proposition 2.2. Let $K \leq L \leq M$ be submodules. Then the following hold.

(1) If $L$ is coneat in $M$, then $L / K$ is coneat in $M / K$.

(2) If $K \leq \operatorname{Rad}(L)$ and $L / K$ is coneat in $M / K$, then $L$ is coneat in $M$. 
(3) If $L \leq M$ is coneat, then $K \leq \operatorname{Rad}(M)$ implies $K \leq \operatorname{Rad}(L)$; hence $\operatorname{Rad}(L)=L \cap \operatorname{Rad}(M)$

(4) If $f: M \rightarrow N$ is a small epimorphism and $L$ is coneat in $M$, then $f(L)$ is coneat in $N$.

(6) If $K$ is coneat in $M$, then $K$ is coneat in $L$ and the converse is true if $L$ is coneat in $M$.

The proof of [20, Lemma A.4] can be adapted to prove the following.

Proposition 2.3. Let $K \leq L \leq M$ be submodules of $M$. If $K$ is coneat in $M$ and $L / K$ is coneat in $M / K$, then $L$ is coneat in $M$.

Proof. Suppose $X$ is a submodule of $L$ such that $L / X$ finitely generated and $L / X$ is small in $M / X$. Firstly we will prove that $K / K \cap X$ is small in $M / K \cap X$.

Assume the contrary. Then there is an $R$-module $W$ such that

$$
K \cap X \leq W \text { and } W+K=M .
$$

Suppose $L /[K+(W \cap X)]$ is not small in $M /[K+(W \cap X)]$. Then there is an $R$-module $Z$ such that $K+(W \cap X) \leq Z$ and $Z+L=M$. Since $K \leq Z, Z=Z \cap W+K$ by (*), and so $M=Z \cap W+L$. By smallness of $L / X$ is small in $M / X, Z \cap W+X=M$. Now $W=Z \cap W+X \cap W$, $W \leq Z$. Finally, since $Z+W=M, Z=M$. Recall that $L / K$ is coneat in $M / K$ and $L /[K+(W \cap X)]$ is epimorphic image of the finitely generated module $L / X$. Hence, $L=K+W \cap X$ by Proposition 2.1(2). By modular law, $X=K \cap X+W \cap X$, and $X \leq W$. Then $K+X=L$. Since $L / X$ is small in $M / X, W=M$ by $(*)$. By our assumption $K$ is coneat in $M$, hence $K=K \cap X$ and $K \leq X$. Since $L / X$ is an epimorphic image of $L / K$ and $L / K$ is coneat in $M / K, \bar{L}=X$ by Proposition 2.1(2), again.

Proposition 2.4 ([15, Lemma 6.1]). Let $A$ be a submodule of an $R$-module $B$ and $i_{A}: A \hookrightarrow B$ be the inclusion map. For a right ideal $I$ of $R, A \cap I B=I A$ if and only if $R / I \otimes A \stackrel{1_{R / I} \otimes i_{A}}{\rightarrow} R / I \otimes B$ is injective.

An exact sequence $0 \rightarrow A \stackrel{f}{\rightarrow} B \rightarrow C$ is said to be coneat exact if $f(A)$ is a coneat submodule of $B$. A monomorphism $f: A \rightarrow B$ is said to be a coneat monomorphism, if the short exact sequence $0 \rightarrow A \stackrel{f}{\rightarrow} B \rightarrow B / f(A) \rightarrow 0$ is coneat exact. Neat-exact sequences are defined in the same manner.

Theorem 2.5. Let $R$ be a commutative ring and $f: N \rightarrow M$ be a monomorphism. The following are equivalent.

(1) $f(N)$ is a coneat submodule of $M$.

(2) $S \otimes_{R} N \stackrel{1_{S} \otimes_{f}}{\rightarrow} S \otimes_{R} M$ is a monomorphism for each simple $R$-module $S$.

(3) $m f(N)=f(N) \cap m M$ for each maximal ideal $m$ of $R$.

Proof. $(1) \Leftrightarrow(2)$ By [8, Proposition 3.1].

$(2) \Leftrightarrow(3)$ Follows by Proposition 2.4. 
Remark 2.6. If $N$ is a pure submodule of $M$, then $N I=N \cap M I$ for every left ideal of $R$ (see, [10, Corollary 4.92]). Therefore, over commutative rings, every pure submodule is coneat by Theorem $2.5(3)$. This fact will be used in the sequel.

Corollary 2.7. Let $R$ be a commutative ring. The following are equivalent.

(1) $0 \rightarrow A \rightarrow B \rightarrow C \rightarrow 0$ is coneat exact.

(2) $0 \rightarrow C^{+} \rightarrow B^{+} \rightarrow A^{+} \rightarrow 0$ is neat exact.

Proof. By Theorem 2.5(2) and the adjoint isomorphism

$$
(M \otimes N)^{+} \cong \operatorname{Hom}\left(M, N^{+}\right) \text {. }
$$

Let $M$ be an $R$-module with $\operatorname{Rad} M=M$. It is easy to see that $\operatorname{Hom}(M, S)=$ 0 for each simple module. Hence,

Corollary 2.8. Let $M$ be a right $R$-module with $\operatorname{Rad}(M)=M$. Then $M$ is absolutely coneat.

A ring $R$ is said to be right small if $R_{R} \ll E\left(R_{R}\right)$. A ring $R$ is small if and only if $E=\operatorname{Rad}(E)$ for every injective $R$-module $E$ (see, [11, Proposition 3.3]).

Proposition 2.9. The following statements are equivalent for a ring $R$.

(1) $R$ is a right small ring.

(2) Absolutely coneat right $R$-modules are precisely those modules $N$ such that $\operatorname{Rad}(N)=N$.

Proof. (1) $\Rightarrow(2)$ Let $E$ be the injective hull of $N$. Then $\operatorname{Rad}(E)=E$ as $R$ is a small ring. Suppose $N$ is coneat in $E$. So that $\operatorname{Rad}(N)=N \cap \operatorname{Rad}(E)=N$ by Proposition 2.2(3). The rest of (2) by Corollary 2.8 .

$(2) \Rightarrow(1)$ Every injective right $R$-module $E$ is absolutely coneat. Then (2) implies $\operatorname{Rad}(E)=E$, and so $R$ is a small ring by [11, Proposition 3.3].

Let $R$ be a ring and $M$ be a nonzero $R$-module. $M$ is called coatomic if every proper submodule $N$ of $M$ is contained in a maximal submodule of $M$, i.e., $\operatorname{Rad}(M / N) \neq 0$.

Proposition 2.10. Let $M$ be a module and $N$ be a coatomic submodule of $M$. Then $N$ is coneat in $M$ if and only if it is coclosed in $M$.

Proof. Suppose $N$ is coneat and $N / X \ll M / X$ for some proper submodule $X \leq N$. Since $N$ is coatomic, $X$ is contained in a maximal submodule, say $K$, of $N$. Then $N / K \ll M / K$, and this contradicts with the fact that $N$ is coneat. Hence $N$ is coclosed. The converse implication is obvious.

In [19], a ring $R$ is called right $K$-ring if every non-zero small right $R$-module is coatomic. Dedekind domains and right max rings (i.e., every nonzero right $R$-module has a maximal submodule) are right $K$-rings. 
Theorem 2.11. $R$ is a right $K$-ring if and only if coneat submodules of any right $R$-module are coclosed.

Proof. For the necessity, let $M$ be a non-zero small module and suppose $M / K$ has no maximal submodules, i.e., $\operatorname{Rad}(M / K)=M / K$ for some proper submodule $K$ of $M$. Then $M / K$ is small and coneat submodule in $E(M / K)$. Hence $M / K$ is coclosed in $E(M / K)$ by (1). This gives a contradiction, since coclosed submodules are not small. Consequently, $K$ is contained in a maximal submodule of $M$, and so $M$ is coatomic.

For the sufficiency, suppose the contrary that, there is a module $M$ and a submodule $N$ of $M$ which is coneat but not coclosed. Then there is a proper submodule $K$ of $N$ such that $N / K \ll M / K$. By Proposition 2.2(1), $N / K$ is a coneat submodule of $M / K$. Then $N / K$ is coatomic by the hypothesis, and so $N / K$ is coclosed by Proposition 2.10 , a contradiction.

\section{Coneat-flat modules}

It is well known that, a right $R$-module $M$ is flat if and only if any short exact sequence of the form $0 \rightarrow K \stackrel{f}{\rightarrow} N \rightarrow M \rightarrow 0$ is pure exact, i.e., $f(K)$ is a pure submodule of $N$. It is natural to ask for which right $R$-modules $P$ any short exact sequence ending with $P$ is coneat exact? In this section several characterizations of such modules are given.

A right $R$-module $M$ is called coneat-flat if the kernel of any epimorphism $Y \rightarrow M \rightarrow 0$ is a coneat submodule of $Y$. Clearly, projective modules are coneat-flat but the converse need not be true in general (see, Theorem 5.1).

Theorem 3.1. The following are equivalent for an $R$-module $M$ :

(1) $M$ is coneat-flat.

(2) $\operatorname{Ext}_{R}^{1}(M, S)=0$ for each simple $R$-module $S$.

(3) There is a coneat exact sequence $0 \rightarrow K \rightarrow L \rightarrow M \rightarrow 0$ with $L$ projective.

(4) There is a coneat exact sequence $0 \rightarrow K \rightarrow L \rightarrow M \rightarrow 0$ with $L$ coneat-flat.

Proof. (1) $\Rightarrow(2)$ Let $\mathbb{E}: 0 \rightarrow S \stackrel{\alpha}{\rightarrow} L \rightarrow M \rightarrow 0$ be a short exact sequence with $S$ simple right $R$-module. Since $M$ is coneat-flat, $S$ is coneat in $L$, and there is a homomorphism $\beta: L \rightarrow S$ such that the following diagram is commutative.

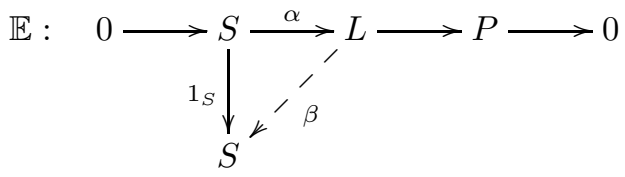

Then $1_{S}=\beta \alpha$, and so the sequence $\mathbb{E}$ splits. Hence $\operatorname{Ext}_{R}^{1}(M, S)=0$.

$(2) \Rightarrow(3)$ Assuming (2). There is a short exact sequence $\mathbb{E}: 0 \rightarrow C \rightarrow F \rightarrow$ $M \rightarrow 0$ with $F$ free $R$-module. Applying $\operatorname{Hom}_{R}(-, S)$, we obtain the exact 
sequence $0 \rightarrow \operatorname{Hom}_{R}(M, S) \rightarrow \operatorname{Hom}_{R}(F, S) \rightarrow \operatorname{Hom}_{R}(C, S) \rightarrow \operatorname{Ext}_{R}^{1}(M, S)=$ 0 .

That is, $\operatorname{Hom}_{R}(\mathbb{E}, S)$ is exact for every simple $R$-module $S$, and so $\mathbb{E}$ is coneat exact.

$(3) \Rightarrow(4)$ is obvious.

$(4) \Rightarrow(1)$ Let $s: B \rightarrow M$ be any epimorphism. Consider the following commutative diagram.

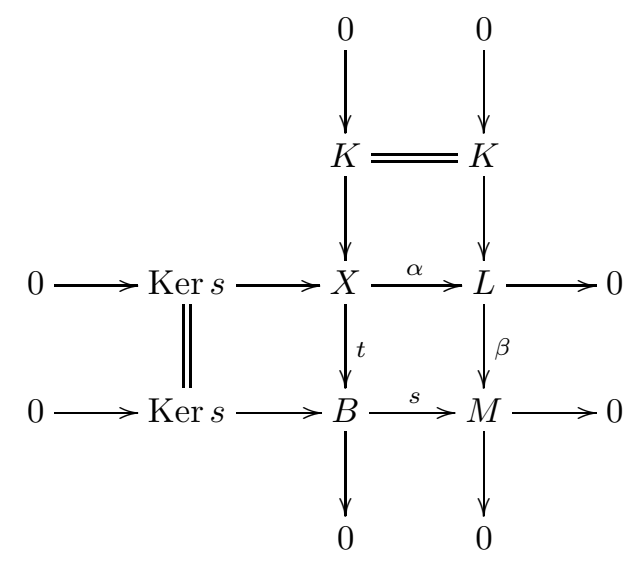

$\beta \alpha=$ st is coneat epimorphism, i.e., $\operatorname{Ker}(s t)$ is a coneat submodule of $X$, by Proposition 2.3. Then $s$ is coneat epimorphism by Proposition 2.2(1). This completes the proof.

By Theorem 3.1, we get the following.

Corollary 3.2. The class of coneat-flat modules is closed under extensions, direct sums, direct summands and coneat quotients. In particular, coneat-flat modules are closed under pure quotients over commutative rings.

Proof. Coneat-flat modules are closed under extensions, direct sums, direct summands and coneat quotients by Theorem 3.1, and under pure quotients by Remark 2.6 and Theorem 3.1.

Proposition 3.3. Let $R$ be a commutative ring and $M$ be an $R$-module. Then $M$ is coneat-flat if and only if $\operatorname{Tor}_{R}(M, S)=0$ for each simple $R$-module $S$.

Proof. Let $0 \rightarrow K \stackrel{i}{\rightarrow} F \rightarrow M \rightarrow 0$ be a short exact sequence with $F$ projective. Applying $-\otimes S$, we get

$$
0=\operatorname{Tor}(F, S) \rightarrow \operatorname{Tor}(M, S) \rightarrow K \otimes S \stackrel{i \otimes 1 S}{\rightarrow} F \otimes S \rightarrow M \otimes S \rightarrow 0 .
$$

Then $i \otimes 1_{S}$ is a monomorphism if and only if $\operatorname{Tor}(M, S)=0$. Now the proof is clear by Theorem 2.5 and Theorem 3.1 .

Proposition 3.4. The following are equivalent for a right $R$-module $M$. 
(1) $M$ is $m$-injective.

(2) $M$ is a neat submodule of an m-injective module.

(3) $M$ is a neat submodule of every module containing it.

(4) $\operatorname{Ext}_{R}^{1}(S, M)=0$ for every simple right $R$-module $S$.

Proof. $(1) \Leftrightarrow(4)$ Let $I$ be a right ideal of $R$. Then applying $\operatorname{Hom}(-, M)$ to the short exact sequence $0 \rightarrow I \stackrel{i}{\rightarrow} R \rightarrow R / I \rightarrow 0$, we get $0 \rightarrow \operatorname{Hom}(R / I, M) \rightarrow$ $\operatorname{Hom}(R, M) \stackrel{i^{*}}{\rightarrow} \operatorname{Hom}(I, M) \rightarrow \operatorname{Ext}^{1}(R / I, M) \rightarrow \operatorname{Ext}_{R}^{1}(R, M)=0$. Then $i^{*}$ is epic if and only if $\operatorname{Ext}_{R}^{1}(R / I, M)=0$.

$(2) \Leftrightarrow(3)$ By [5, Theorem 3.3].

$(3) \Leftrightarrow(4)$ By $[5$, Theorem 3.4. (i) $\Leftrightarrow($ ii) $]$.

Proposition 3.5. Let $R$ be a commutative ring. An $R$-module $M$ is coneat-flat if and only if $M^{+}$is m-injective.

Proof. Let $S$ be a simple $R$-module. We have the standard isomorphism

$$
\operatorname{Ext}_{R}^{1}\left(S, M^{+}\right) \cong \operatorname{Tor}_{1}^{R}(M, S)^{+} .
$$

Now, the proof is immediate by Proposition 3.3 and Proposition 3.4.

Corollary 3.6. Let $R$ be a commutative ring. The class of coneat-flat modules is closed under pure submodules.

Proof. Let $0 \rightarrow A \rightarrow B \rightarrow C \rightarrow 0$ be a pure exact sequence of $R$-modules with $B$ coneat-flat. Then the short exact sequence $0 \rightarrow C^{+} \rightarrow B^{+} \rightarrow A^{+} \rightarrow 0$ splits. By Proposition 3.5 the module $B^{+}$is $m$-injective, and so $A^{+}$is $m$-injective. Then $A$ is coneat-flat by Proposition 3.5, again.

Proposition 3.7. The following statements are equivalent for a ring $R$.

(1) $R$ is a right $V$-ring.

(2) for every right $R$-module $M$ every submodule of $M$ is coneat in $M$.

(3) every right $R$-module is coneat-flat.

Proof. $(1) \Rightarrow(2)$ is clear, since every simple right $R$-module is injective by (1).

$(2) \Rightarrow(3)$ Let $M$ be a right $R$-module. Consider an epimorphism $f: F \rightarrow M$ with $F$ free right $R$-module. Then $\operatorname{Ker} f$ is a coneat submodule of $F$ by (2). Therefore $M$ is coneat-flat by Theorem 3.1 .

$(3) \Rightarrow(1)$ Let $S$ be a simple $R$-module and $E$ be an injective module containing $S$. By the hypothesis $E / S$ is coneat-flat. Hence the sequence $0 \rightarrow S \rightarrow E \rightarrow E / S \rightarrow 0$ splits by Theorem 3.1, and so $S$ is injective.

\section{When coneat-flat modules are flat}

In this section, we study the flatness of coneat-flat modules, and the character of coneat-flat modules. We begin with the following. A module right $R$-module $M$ is called cotorsion if $\operatorname{Ext}_{R}^{1}(F, M)=0$ for any flat $R$-module $F$. 
Example 4.1. (1) Let $R$ be a valuation domain with a non finitely generated maximal ideal $P$. Then $\operatorname{Rad}(P)=P^{2}=P$, and so $P$ is a coneat submodule of $R$ by Corollary 2.8. Hence $R / P$ is coneat-flat by Theorem 3.1. On the other hand, $R / P$ is a not a flat $R$-module, since $R / P$ is a torsion $R$-module.

(2) Let $R$ be a regular ring that is not a right $V$-ring. Then there exists a flat module which is not coneat-flat by Proposition 3.7.

In light of Example 4.1, it is natural to consider the rings over which coneatflat and flat modules coincide. We begin with the following lemma.

Lemma 4.2. Let $R$ be a ring and $S$ be a simple $R$-module. If $R$ is commutative or semilocal, then $S$ is cotorsion.

Proof. First suppose $R$ is commutative and let $I=A n n_{R}(S)$. Then clearly $S$ is an $R / I$-module. Since $R / I$ is simple, $S$ is cotorsion as an $R / I$-module. So that $S$ is a cotorsion $R$-module by [18, Proposition 3.3.3]. If $R$ is semilocal, then $J(R) . S=0$ and so $S$ is an $R / J(R)$-module. As $R$ is semilocal, $R / J(R)$ is semisimple and so $S$ is a cotorsion $R / J(R)$-module. Now, $S$ is a cotorsion $R$-module by [18, Proposition 3.3.3], again.

Corollary 4.3. Suppose $R$ is commutative or a semilocal ring. Then every flat module is coneat-flat.

Proof. Let $S$ be a simple $R$-module. Then $S$ is a cotorsion module by Lemma 4.2. Therefore $\operatorname{Ext}_{R}^{1}(M, S)=0$, and so $M$ is coneat-flat by Theorem 3.1.

Remark 4.4. A commutative domain $R$ is called almost perfect if $R / I$ is a perfect ring for each nonzero ideal $I$ of $R$. It is clear that almost perfect domains are $C$-rings. In [14], the authors prove that, if $R$ is an almost perfect domain, then an $R$-module $M$ is injective if and only if $\operatorname{Ext}_{R}^{1}(S, M)=0$ (i.e., $M$ is $m$-injective) for each simple module $S$. Actually, one of the characterization of right $C$-rings is the following: $R$ is a right $C$-ring if and only if every $m$ injective right $R$-module is injective (see, [16, Lemma 4]).

Proposition 4.5. Let $R$ be a left $C$-ring. A right $R$-module $M$ is flat if and only if $\operatorname{Tor}_{1}^{R}(M, S)=0$ for each simple left $R$-modules $S$.

Proof. Necessity is clear. For the sufficiency assume that $\operatorname{Tor}_{1}^{R}(M, S)=0$ for each simple left $R$-modules $S$. Then $0=\operatorname{Tor}_{1}^{R}(M, S)^{+} \cong \operatorname{Ext}_{R}^{1}\left(S, M^{+}\right)$implies $M^{+}$is $m$-injective by Theorem 3.4. Therefore $M^{+}$is injective, because $R$ is a left $C$-ring. Hence $M$ is flat by [7, Theorem 3.2.10].

Proposition 4.6. Let $R$ be a commutative ring. Consider the following statements.

(1) $R$ is a $C$-ring.

(2) Coneat-flat $R$-modules are flat.

Then $(1) \Rightarrow(2)$. If $R$ is a noetherian, then $(2) \Rightarrow(1)$. 
Proof. (1) $\Rightarrow(2)$ By Corollary 3.3 and Proposition 4.5.

$(2) \Rightarrow(1)$ Let $M$ be an $m$-injective $R$-module. Then $M^{+}$is flat by the hypothesis and Theorem 4.10. As $R$ is noetherian, $M$ is injective by [3, Theorem $2]$. Hence $R$ is a $C$-ring.

Theorem 4.7. The following are equivalent for a commutative ring $R$.

(1) Every coneat-flat module is flat.

(2) Flat modules are precisely those modules $M$ satisfying

$$
\operatorname{Ext}^{1}\left(M, \prod_{i \in I} S_{i}\right)=0
$$

where the $S_{i}$ 's are all the non-isomorphic simple modules.

Proof. $(1) \Rightarrow(2)$ By Lemma 4.2, simple modules are cotorsion. Then $\prod_{i \in I} S_{i}$ is cotorsion, since cotorsion modules are closed under direct products. Hence, if $M$ is flat, then $\operatorname{Ext}_{R}^{1}\left(M, \prod_{i \in I} S_{i}\right)=0$. Conversely, suppose $\operatorname{Ext}_{R}^{1}\left(M, \prod_{i \in I} S_{i}\right)$ $=0$. Then $\operatorname{Ext}_{R}^{1}\left(M, S_{i}\right)=0$ for each $i \in I$. So that $M$ is coneat-flat by Theorem 3.1. Hence $M$ is flat by (1).

$(2) \Rightarrow(1)$ Suppose $M$ is coneat-flat. Then $\operatorname{Ext}_{R}^{1}(M, S)=0$ for each simple $R$-module $S$. So that $\operatorname{Ext}_{R}^{1}\left(M, \prod_{i \in I} S_{i}\right)=0$ for any index set $I$ and simple $R$-modules $S_{i}$. Hence $M$ is flat by (2).

Proposition 4.8. Let $R$ be a commutative $N$-ring and $M$ be an arbitrary $R$-module. Then the following hold.

(1) $M$ is $m$-injective if and only if $M^{+}$is coneat-flat.

(2) $M$ is $m$-injective if and only if $M^{++}$is $m$-injective.

(3) $M$ is coneat-flat if and only if $M^{++}$is coneat-flat.

(4) Any direct product of coneat-flat modules is coneat-flat.

(5) Any direct product of copies of $R$ is coneat-flat.

(6) The class of m-injective modules is closed under pure quotients.

Proof. (1) An $R$-module $M$ is $m$-injective module if and only if $M^{+}$is coneatflat by [13, Theorem 9.51], since $R$ is an $N$-ring

(2) $M$ is $m$-injective if and only if $M^{+}$is coneat-flat by (1), and $M^{+}$is coneat-flat if and only if $M^{++}$is $m$-injective by Proposition 3.5.

(3) If $M$ is coneat-flat, then $M^{+}$is $m$-injective by Proposition 3.5 . So $M^{+++}$is $m$-injective by $(2)$, and hence $M^{++}$is coneat-flat. Conversely, if $M^{++}$is coneat-flat, then $M$ is coneat-flat by Corollary 3.6, since $M$ is a pure submodule of $M^{++}$.

(4) Let $\left(M_{i}\right)_{i \in J}$ be a family of coneat-flat $R$-modules. Since the class of coneat-flat modules is closed under direct sums, $\bigoplus_{i \in J} M_{i}$ is coneat-flat. So $\left(\bigoplus M_{i}\right)^{++} \cong\left(\prod M_{i}^{+}\right)^{+}$is coneat-flat by (3). Since $\bigoplus_{i \in J} M_{i}^{+}$is a pure submodule of $\prod_{i \in J} M_{i}^{+},\left(\oplus_{i \in J} M_{i}^{+}\right)^{+}$is a direct summand of $\left(\prod_{i \in J} M_{i}^{+}\right)^{+}$, and so $\left(\oplus_{i \in J} M_{i}^{+}\right)^{+} \cong \prod_{i \in J} M_{i}^{++}$is coneat-flat. Since coneat-flat modules are closed 
under pure submodules and $\prod_{i \in J} M_{i}$ is a pure submodule of $\prod_{i \in J} M_{i}^{++}$, the module $\prod_{i \in J} M_{i}$ is coneat-flat.

(5) By (4).

(6) Take any pure exact sequence $0 \rightarrow A \rightarrow B \rightarrow C \rightarrow 0$ with $B m$-injective. Then we have a split exact sequence $0 \rightarrow C^{+} \rightarrow B^{+} \rightarrow A^{+} \rightarrow 0$. By (1), $B^{+}$is coneat-flat, and so $C^{+}$is coneat-flat. Then $C$ is $m$-injective by (1), again.

An $R$-module $M$ is called absolutely pure if it is pure in every module containing it as a submodule. It is well known that, a ring $R$ is left noetherian if and only if every absolutely pure left $R$-module is injective.

Proposition 4.9. $R$ is a left $N$-ring if and only if every absolutely pure left $R$-module is m-injective.

Proof. $(\Rightarrow)$ Let $M$ be an absolutely pure left $R$-module. Since $R$ is a left $N$-ring, $\operatorname{Ext}_{R}^{1}(S, M)=0$ for each simple left $R$-module $S$. That is, $M$ is $m$-injective.

$(\Leftarrow)$ Let $S$ be a simple left $R$-module. Then $\operatorname{Ext}_{R}^{1}(S, M)=0$ for each absolutely pure left $R$-module $M$ by the assumption. Then $S$ is finitely presented by $[6$, Proposition $]$.

Theorem 4.10. Let $R$ be a ring. The following statements are equivalent.

(1) (a) $M$ is a flat right $R$-module if and only if $\operatorname{Tor}_{1}^{R}(M, S)=0$ for each simple left $R$-module $S$,

(b) $R$ is a left $N$-ring.

(2) $M$ is an $m$-injective left $R$-module if and only if $M^{+}$is flat.

(3) $M$ is an $m$-injective left $R$-module if and only if $M$ is an absolutely pure left $R$-module.

Proof. (1) $\Rightarrow(2)$ Let $M$ be a left $R$-module and $S$ be a simple left $R$-module. Suppose $M$ is $m$-injective. Then $0=\operatorname{Ext}_{R}^{1}(S, M)^{+} \cong \operatorname{Tor}_{1}^{R}\left(M^{+}, S\right)$ by $[13$, Theorem 9.51], and so $M^{+}$is flat by (1). Conversely suppose $M^{+}$is flat. Then $M^{++}$is injective by [13, Theorem 3.52], and so $M$ is absolutely pure, since $M$ is pure in $M^{++}$. Therefore $M$ is $m$-injective by Proposition 4.9.

$(2) \Rightarrow(3)$ Firstly, we shall prove that a right $R$-module $M$ is flat if and only if $M^{++}$is flat. Then $R$ is left coherent by [3, Theorem 1]. Suppose $M$ is a flat right $R$-module. Then $M^{+}$is $\left(m\right.$-)injective, and so $M^{++}$is flat by $(2)$. Now, conversely suppose $M^{++}$is a flat right $R$-module. Then $M$ is flat, since $M$ is pure submodule of $M^{++}$and flat modules closed under pure submodules.

Let $M$ be a left $R$-module. Then $M^{+}$is flat if and only if $M$ is absolutely pure by [3, Theorem 1], since $R$ is left coherent. Hence the rest of (3) follows by $(2)$.

$(3) \Rightarrow(1)$ Suppose $\operatorname{Tor}_{1}^{R}(M, S)=0$ for each simple left $R$-module $S$. Then $\operatorname{Ext}_{R}^{1}\left(S, M^{+}\right)=0$, and so $M^{+}$is m-injective. Then $M^{+}$is absolutely pure by (3). Therefore $M^{+}$is injective, since it is pure-injective. Thus $M$ is flat. This proves (a), and (b) follows by Proposition 4.9. 
Proposition 4.11. Let $R$ be a commutative ring. Consider the following statements.

(1) $R$ is a $C$-ring.

(2) Coneat-flat $R$-modules are flat.

Then $(1) \Rightarrow(2)$. If $R$ is a noetherian, then $(2) \Rightarrow(1)$.

Proof. (1) $\Rightarrow(2)$ By Proposition 3.3 and Proposition 4.5.

$(2) \Rightarrow(1)$ Let $M$ be an $m$-injective $R$-module. Then $M^{+}$is flat by the hypothesis and Theorem 4.10. As $R$ is noetherian, $M$ is injective by [3, Theorem 2]. Hence $R$ is a $C$-ring.

It is easy to see that, a left $N$-ring and left semiartinian ring is left noetherian. The following is a slight generalization of this fact.

Corollary 4.12. If $R$ is a left $N$-ring and a left $C$-ring, then $R$ is left noetherian.

Proof. By Proposition 4.5 and Theorem 4.10, a left $R$-module $M$ is $m$-injective if and only if it is absolutely pure. So that every absolutely pure left module is injective. Hence $R$ is left noetherian.

Note that, Corollary 4.12, generalizes [5, Theorem 4.1 (ii) $\Rightarrow(\mathrm{i})]$.

In [3, Theorem 4], the authors proves that, $R$ is left artinian if and only if a left module $M$ is injective exactly when $M^{+}$is projective. We show that, this result still holds if we replace $m$-injective by injective.

Theorem 4.13. Let $R$ be a ring. The following are equivalent.

(1) $R$ is left artinian.

(2) A left $R$-module $M$ is $m$-injective if and only if $M^{+}$is projective.

Proof. (1) $\Rightarrow(2) R$ is a left $C$-ring by (1), and so $m$-injective modules are injective. Now, (2) follows by [3, Theorem 4].

$(2) \Rightarrow(1)$ Firstly, we show that a left $R$-module $M$ is $m$-injective if and only if $M$ is absolutely pure.

Let $M$ be an absolutely pure left $R$-module. Consider the pure exact sequence $0 \rightarrow M \rightarrow E(M) \rightarrow E(M) / M \rightarrow 0$. Then the short exact sequence $0 \rightarrow(E(M) / M)^{+} \rightarrow E(M)^{+} \rightarrow M^{+} \rightarrow 0$ splits. Then $E(M)^{+}$is projective, and hence $M^{+}$is projective. By (2), $M$ is $m$-injective. Conversely, let $M$ be an $m$-injective left $R$-module. Since $M$ is pure in $M^{++}$and $M^{++}$is injective, $M$ is absolutely pure.

Then a left $R$-module $M$ is $m$-injective if and only if $M$ is absolutely pure if and only if $M^{+}$is projective. By [3, Theorem 3], $R$ is right perfect, and so it is a left $C$-ring, i.e., $m$-injective left $R$-modules are injective. Hence $R$ is left artinian by [3, Theorem 4] and (2). 


\section{When coneat-flat modules are projective}

In this section, we shall consider when coneat-flat modules are projective. We begin with the following result.

Theorem 5.1. Consider the following statements.

(1) $R$ is a right perfect ring.

(2) Every coneat-flat right $R$-module is projective.

Then $(1) \Rightarrow(2)$. If $R$ is either commutative or semilocal, then $(2) \Rightarrow(1)$.

Proof. $(1) \Rightarrow(2)$ Let $P$ be a coneat-flat module. Consider a short exact sequence $0 \rightarrow K \rightarrow F \rightarrow P \rightarrow 0$ with $F$ free module. Since $R$ is perfect, $F$ is supplemented by $[17,43.9]$. So $K$ has a supplement in $F$, that is, $K+N=F$ and $A \cap N \ll N$ for some submodule $N$ of $F$. On the other hand, $K$ is coatomic, as $R$ is a perfect ring. Then $K$ is a coclosed submodule of $F$ by Proposition 2.10. So that $K \cap N \ll K$. Hence $K$ and $N$ are mutual supplements, and so $K \oplus N=F$ by $[17,41.15]$. Therefore $N \cong F / K \cong P$ is projective.

$(2) \Rightarrow(1)$ Let $M$ be a flat module. By Corollary 4.3, $M$ is coneat-flat, and so $M$ is projective by (2). Hence $R$ is a perfect ring.

The following is an immediate consequence of Theorem 5.1.

Corollary 5.2. Let $R$ be a perfect ring. Then an $R$-module $P$ is projective if and only if $\operatorname{Ext}_{R}^{1}(P, S)=0$ for every simple $R$-module $S$.

An epimorphism $f: N \rightarrow M$ is said to be a small cover of $M$ if $\operatorname{Ker} f \ll N$. Moreover, if $N$ is projective, then $f$ is called a projective cover.

Proposition 5.3. Let $R$ be a ring and $M$ be a right $R$-module with a projective cover $f: P \rightarrow M$. Set $K=\operatorname{Ker} f$. Then $M$ is a coneat-flat module if and only if $\operatorname{Rad}(K)=K$.

Proof. $(\Rightarrow)$ Assume $\operatorname{Rad}(K) \neq K$. Then $K$ has a maximal submodule, say $A$. By Proposition 2.1, there exists a maximal submodule $L$ of $P$ such that $A=K \cap L$. Then $K \leq \operatorname{Rad} P$ implies $K=K \cap \operatorname{Rad}(P) \leq K \cap L=A$. Contradiction. Hence (2) holds.

$(\Leftarrow)$ By Corollary 2.8 and Theorem 3.1.

Corollary 5.4. Let $R$ be a semiperfect ring. Then finitely presented coneat-flat modules are projective.

Lemma 5.5. Let $R$ be a commutative ring and $M$ be a coneat-flat $R$-module. Then, for all maximal ideals $m$ of $R, M_{m}$ is a coneat-flat $R_{m}$-module.

Proof. Since $M$ is a coneat-flat $R$-module, there is a short exact sequence $0 \rightarrow$ $K \rightarrow F \rightarrow M \rightarrow 0$ where $K$ is coneat submodule of $F$ with $F$ is a projective $R$-module by Theorem 3.1. By exactness of localization, for all maximal ideals $m$ of $R$, the sequence $0 \rightarrow K_{m} \rightarrow F_{m} \rightarrow M_{m} \rightarrow 0$ is exact. Since $m K=K \cap m F$ 
for all maximal ideals $m$ of $R$, we have $m_{m} K_{m}=K_{m} \cap m_{m} F_{m}$. Therefore $M_{m}$ is a coneat-flat $R_{m}$-module by Theorem 2.5 .

Corollary 5.6. Let $R$ be a commutative ring. Then a finitely presented $R$ module $M$ is coneat-flat if and only if it is projective.

Proof. Sufficiency is clear. For the necessity, suppose $M$ is coneat-flat. Let $m$ be a maximal ideal of $R$. Then $M_{m}$ is a coneat-flat $R_{m}$-module by Lemma 5.5 . So that $M_{m}$ is projective (and so flat) over $R_{m}$ by Corollary 5.4. Then $M$ is flat by [10, page 160, Exercise 14]. Therefore $M$ is projective by [10, Theorem 4.30].

\section{References}

[1] E. Büyükaşık and Y. Durğun, Neat-flat modules, http://arxiv.org/abs/1306.2860.

[2] Absolutely s-pure modules and neat-flat modules, Comm. Alg. 43 (2015), no. $2,384-399$.

[3] T. J. Cheatham and D. R. Stone, Flat and projective character modules, Proc. Amer. Math. Soc. 81 (1981), no. 2, 175-177.

[4] J. Clark, C. Lomp, N. Vanaja, and R. Wisbauer, Lifting Modules, Frontiers in Mathematics, Basel, 2006.

[5] S. Crivei, Neat and coneat submodules of modules over commutative rings, Bull. Aust. Math. Soc. 89 (2014), no. 2, 343-352.

[6] E. Enochs, A note on absolutely pure modules, Canad. Math. Bull. 19 (1976), no. 3, $361-362$.

[7] E. E. Enochs and O. M. G. Jenda, Relative Homological Algebra, Walter de Gruyter \& Co., Berlin, 2000.

[8] L. Fuchs, Neat submodules over integral domains, Period. Math. Hungar. 64 (2012), no. 2, 131-143.

[9] A. I. Generalov, Weak and $\omega$-high purities in the category of modules, Mat. Sb. (N.S.) 105(147) (1978), no. 3, 389-402, 463.

[10] T. Y. Lam, Lectures on Modules and Rings, Springer-Verlag, New York, 1999.

[11] C. Lomp, On the splitting of the dual Goldie torsion theory, Algebra and its applications (Athens, OH, 1999), 377-386, Contemp. Math., 259, Amer. Math. Soc., Providence, RI, 2000.

[12] G. Renault, Étude de certains anneaux liés aux sous-modules compléments d'un Amodule, C. R. Acad. Sci. Paris 258 (1964), 4888-4890.

[13] J. Rotman, An Introduction to Homological Algebra, Academic Press Inc., New York, 1979.

[14] L. Salce, Almost perfect domains and their modules, Commutative algebra-Noetherian and non-Noetherian perspectives, 363-386, Springer, New York, 2011.

[15] E. G. Skljarenko, Relative homological algebra in the category of modules, Uspehi Mat. Nauk 33 (1978), no. 3(201), 85-120.

[16] P. F. Smith, Injective modules and prime ideals, Comm. Algebra 9 (1981), no. 9, 989999.

[17] R. Wisbauer, Foundations of Module and Ring Theory, Gordon and Breach, Reading, 1991.

[18] J. Xu, Flat Covers of Modules, Springer-Verlag, Berlin, 1996.

[19] H. Zöschinger, Koatomare Moduln, Math. Z. 170 (1980), no. 3, 221-232.

[20] _ Schwach-injektive Moduln, Period. Math. Hungar. 52 (2006), no. 2, 105-128.

[21] _ Schwach-Flache Moduln, Comm. Algebra 41 (2013), no. 12, 4393-4407. 
ENGIN BÜYÜKAŞIK

IZMIR Institute of TeChNology

Department of Mathematics

35430, İMIIR, TURKEY

E-mail address: enginbuyukasik@iyte.edu.tr

Yilmaz DuRĞUN

BITTLIS EREN UNIVERSITY

Department of Mathematics

13000, BİTLIS, TURKEY

E-mail address: ydurgun@beu.edu.tr 